\title{
Underlying gauge-fermion models of compositeness
}

\author{
Gabriele Ferretti ${ }^{1, a}$ \\ ${ }^{1}$ Department of Physics, Chalmers University of Technology, Fysikgården, 41296 Göteborg, Sweden
}

Received 7 October 2020 / Accepted 7 July 2021 / Published online 19 July 2021

(C) The Author(s) 2021

\begin{abstract}
We review recent advances in the construction of models of compositeness using four-dimensional gauge theories with fermionic matter. We discuss the group theory data needed to fulfill the basic phenomenological requirements and we touch upon the main predictions of these constructions.
\end{abstract}

\section{Introduction}

The most obvious way of solving the conundrum arising by the presence of a light spin zero excitation is to do away with scalar fields altogether at the fundamental level. This idea was among the very first things that were tried [1-3] in going beyond the standard model (BSM) and had many different names [Technicolor (TC), Hypercolor (HC)...] and incarnations [4,5]. See [6] and references therein for reviews of the early models of this kind. Even earlier, in [7], a model where the vector bosons are also composite had been proposed.

Many of the original models did not survive the ensuing tests from LEP, LHC, and other experiments. Obviously, all "Higgless" models are now ruled out, as are models predicting excessive flavor changing neutral processes, CP violation, or correction to Electroweak precision parameters. However, at the price of some finetuning, one can still envisage many scenarios that pass the above experimental constraints. One such class of models is characterized by having the Higgs boson arising as a (pseudo)-Nambu-Goldstone boson (pNGB) of a spontaneously broken global symmetry (SSB) [8] thus explaining its lightness. These models are often broadly referred to as composite Higgs models $(\mathrm{CH})$ in the literature, although one should keep in mind that there are also models where the Higgs is a composite particle without being a pNGB. In the following discussion, we only consider the case of the Higgs as a pNGB. Although we shall not review the details here (see, e.g., [9] and references therein), there is a subclass of $\mathrm{CH}$ models, referred to as "little Higgses" (LH) in which the symmetry breaking required to generate the Higgs mass occurs when at least two couplings with the SM are nonzero, mitigating the fine-tuning problem by pushing the quadratic divergences to higher loops.

\footnotetext{
a e-mail: ferretti@chalmers.se (corresponding author)
}

\section{Underlying gauge-fermion models}

We concentrate on purely four-dimensional realizations of SSB in a $\mathrm{CH}$ model arising from a gauge theory containing only fermionic matter that becomes strongly coupled in the infra-red (IR).

Let us first briefly recall how SSB of a global symmetry group $G$ to a subgroup $H$ is realized via condensation of a fermionic bilinear. We work with lefthanded (LH) Weyl fermions and think of a Dirac fermion as a pair of LH fermions with conjugate quantum numbers. The fermions transform under some vector-like representation of a hypercolor group $G_{\mathrm{HC}}$. The three fundamental patterns of SSB $G / H$ are: [1012] $S U(N) / S O(N)$, for $N$ fermions $\psi_{i}$ in a real irreducible representation (irrep.); $S U(2 N) / S p(2 N)$ for $2 N$ fermions in a pseudo-real irrep. and lastly $S U(N) \times$ $S U(N) / S U(N)$ for $N$ fermion pairs $\left(\psi_{i}, \tilde{\psi}^{i}\right)$ in a complex irrep. The unbroken symmetry group $H$ is understood as the subgroup of $G$ leaving invariant the vacuum condensate $\left\langle\psi_{i} \psi_{j}\right\rangle$ in the first two cases or $\left\langle\psi_{i} \tilde{\psi}^{j}\right\rangle$ in the third one.

At this stage, the actual embedding of $H$ into $G$ is still arbitrary and $H$ can be "rotated" inside $G$ by conjugation with an element of $G$ by acting appropriately on the condensate $\left(H \rightarrow g H g^{T}\right.$ or $\left.H \rightarrow g H g^{\dagger}\right)$. Equivalently, we can think of having turned the vacuum condensate into a non-linear field parameterized by angular-valued fields corresponding to the broken directions. The fact that all directions are equivalent corresponds to the fact that no potential is yet present for these angular fields.

A privileged reference can only be obtained by explicitly breaking the overall $G$ symmetry and this is what happens when introducing bare masses or couplings to the SM. We want to gauge part of the $G$ symmetry in such a way that the $W^{ \pm}$and $Z$ gauge bosons acquire a mass, leaving unbroken a "custodial" global $S U$ (2) group [13], but also leaving at least a set of pNGBs with the quantum numbers of the SM Higgs 
field [14]. This singles out the following minimal cosets among those in [15] that can be realized with hyperfermions: $S U(5) / S O(5), S U(4) / S p(4)$, and $S U(4) \times$ $S U(4) / S U(4)$.

Larger cosets are also of interest in LH constructions or because some of the remaining weakly coupled pNGBs could play the role of Dark Matter (discussed elsewhere in this review). Dynamically, the privileged direction is realized by the generation of a potential for the angular variables fixing their value at a minimum.

Typically, one considers only one non-zero direction parameterized by the Higgs vacuum expectation value (vev) $v=246 \mathrm{GeV}: \sin \theta=v / f$, with $f$ being the pNGB decay constant, but in general, there can be more than one custodial directions. In phenomenological studies, the value of $\theta$ (or equivalently $f$ ) is kept arbitrary and varied in the region still allowed by, e.g., precision tests, but it is important to keep in mind that, once we break the symmetry, $\theta$ is no longer a "modulus" and it is fixed by the underlying dynamics, computable on the lattice (discussed elsewhere in this review).

In the limit $\theta \rightarrow 0$ (keeping $f$ fixed) the masses of all SM gauge bosons vanish and the full EW symmetry is restored, i.e., aligned with some of the directions in $H$. The $\theta \neq 0$ case is then referred to as vacuum "misalignment". The opposite extreme $\theta \rightarrow \pi / 2$ is referred to as the TC limit.

It is not straightforward to generate a vacuum misalignment, since the SM gauge interactions by themselves are not sufficient $[10,11,16]$. An early way to achieve this in the context of a $S U(5) / S O(5)$ theory was proposed in [17] by adding an additional $U(1)$ interaction whose gauge boson has a different agenda and pulls the vacuum in another direction. A similar coset arises in the little Higgs construction of [18]. In this case, however, the need to implement collective symmetry breaking by gauging two copies of the EW group requires enlarging the coset to $S U(7) / S O(7)$ if one wants to consider fermionic realizations.

A similar little Higgs construction based on a pseudoreal irrep is given in [19]. Here, one needs to consider at least the enlarged cosets $S U(6) / S p(6)$ in order for collective SSB to work in a fermionic realization. The original "Minimal moose" construction [20] would require even more hyperfermions in a complex irrep. We will not discuss fermionic realizations of the Minimal moose, but a model based on complex irreps, closer in spirit to the idea of this section is found in [21]. Two more fermionic realizations trying to account for the recent constraints, where the top plays a crucial dynamical role, can be found in $[22,23]$.

Models with additional gauge bosons are now severely constrained by direct searches, so one is led to consider alternatives to [17]. Fermions come to the rescue $[18,24,25]$, since their couplings can naturally provide the required misaligning contribution to the potential. The top quark is the prime candidate for the job, since it is the SM fermion that couples most strongly to the Higgs sector.

The proposal [25] was framed in the context of a fivedimensional theory defined on a slice of AdS space.
This description is seen as the holographic dual of a strongly coupled theory giving rise to the simplest coset preserving custodial symmetry, namely $S O(5) / S O(4)$. (Note that this coset is not among those obtained from the gauge theories considered in this work.) The two four-dimensional boundaries are known as the IR and UV brane and the mass hierarchy is explained by the "warp" factor in the AdS metric.

The Higgs field is identified with the zero mode of the fifth component of a $S O(5)$ bulk gauge field $A_{5}$ which is protected from acquiring a mass at tree level by gauge invariance. The bulk fermions decompose into zero modes (present only for some specific boundary conditions) and higher modes. The physical fermions arise from the mixing of these modes via a term localized at the IR brane. The mass of the lowest lying physical fermions is then generated by a (pre-)Yukawa interaction of the Higgs field with the zero mode and the higher modes. This mechanism, alternative to a direct generation of fermion masses via a bilinear coupling of the Higgs to the zero modes, goes under the name of partial compositeness (PC).

If one does not take the five-dimensional approach, one can still generate a mass via PC by coupling to composite states made of trilinears in the hyperfermions. This was actually the very first realization of the PC mechanism proposed by Kaplan in his seminal paper [26]. In this case, there is no real need to use the same mechanism for all fermions, and one can envisage a situation where only the heaviest fermions have a partner, alleviating the difficulties with the top quark mass without adding additional dof. This compromise solution is also used in the LH constructions $[18,19]$.

\section{Partial compositeness}

The recent developments on which this section is concerned are attempts at combining the $\mathrm{CH}$ and $\mathrm{PC}$ ideas $[8,26]$ in a four-dimensional setting. We identify the allowed underlying hypercolor groups and sketch their main properties.

The question of what requirements one should fulfill at the group theory level was addressed in [27]. At the same time, a particular example of such models was constructed [28] and studied in details. More models along those lines followed, e.g., $[29,30]$. We start by reviewing the basic ingredients of such constructions. It should be clear that nothing prevents extending these simple considerations to the LH type of constructions.

To establish a self-contained class of representative models of this sort, one is forced to make further simplifying assumptions. Perhaps, the most basic one is that the underlying gauge theory be based on a simple hypercolor group $G_{\mathrm{HC}}$. This is certainly not a physical requirement and it might even be necessary to drop it if one wants to proceed toward more complete theories where one finds an explanation of all the required fourfermi couplings or a mechanism to solve the strong $\mathrm{CP}$ problem, as recently proposed in [31]. It seems however 
reasonable to assume that the next step beyond the SM be dominated by one such gauge interaction and focus on its dynamics as a start.

Assuming having chosen a hypercolor group $G_{\mathrm{HC}}$, the fermionic matter content is now limited by physical considerations. To begin with, we want to have sufficient number of fields to realize the $\mathrm{CH}$ cosets above but not too many fields as to loose asymptotic freedom, since we want the theory to go to strong coupling in the IR. This in itself is not a difficult task. It becomes tighter when we add $\mathrm{PC}$ and also require the possible formation of fermionic bound states with the SM quantum numbers of at least the third family quarks $Q_{L}^{3}=\left(t_{L}, b_{L}\right)$ and $u_{R}^{3}=t_{R}$. This is the minimum amount of composite fermions necessary for $\mathrm{PC}$ to generate the top quark mass, the partner to the LH bottom field being required to avoid breaking weak isospin.

The construction of top partners requires some of the hyperfermions to carry color charge, thus enlarging the group of global symmetries of the hypercolor theory to make room for QCDs $S U(3)$ as well as the EW group. There are essentially two ways to do this. The first alternative is to have just more of the same flavors of fermions and enlarge the minimal cosets to larger ones $S U(N) / S O(N), S U(2 N) / S p(2 N)$ and $S U(N) \times S U(N) / S U(N)$ where $N$ is chosen, so that $S U(3)$ also fits in the unbroken subgroup. (If one does not require the type of cosets arising in the underlying gauge-fermi theory constructions, one can also consider cosets such as $S O(11) / S O(10)[32]$.

The case of fermions only in a pseudo-real irrep of $G_{\mathrm{HC}}$ (yielding $S U(2 N) / S p(2 N)$ ) is ruled out, since three pseudo-scalars cannot combine to a singlet to form top partners. This coset can be rescued by introducing one additional hyperfermion in a real irrep whose main purpose is to allow the construction of trilinears [33].

The case of a real irrep. formally admits the minimum solutions $G_{\mathrm{HC}}=G_{2}$ with $11 \psi \in \mathbf{7}$ and $G_{\mathrm{HC}}=F_{4}$ with $11 \psi \in \mathbf{2 6}$, both yielding the coset $S U(11) / S O(11)$ $(S U(10) / S O(10)$ does not give rise to a Higgs pNGB with the right quantum numbers). This solution might be of academic interest only, since it is difficult to prevent proton decay without further ad hoc assumptions.

As for the complex irreps, [30] showed that the minimal coset $S U(7) \times S U(7) / S U(7)$ is viable and it is possible to implement baryon number conservation. The coset can also be easily enlarged to $N>7$ if one wants to generate partners to all SM fermions. The requirement that it be possible to construct hypercolor singlets by combining three fermions in the same complex irrep singles out three solutions for $G_{\mathrm{HC}}$. One is the solution presented in [30] $G_{\mathrm{HC}}=S U(3)$ with $\psi \in \mathbf{3}$, using the same invariant $\epsilon^{a b c}$ employed in ordinary QCD. Additionally, one could consider $G_{\mathrm{HC}}=S U(6)$ with $\psi \in \mathbf{1 5}$ (the two-index antisymmetric, using the invariant $\left.\epsilon^{a_{1} \ldots a_{6}}\right)$ and $G_{\mathrm{HC}}=E_{6}$ with $\psi \in \mathbf{2 7}$ and the standard trilinear invariant of $E_{6}$.

A second alternative is to employ a different type of fermion $\chi$ (and $\tilde{\chi}$ if complex) to carry color. By this, we mean that the two types of fermions $\psi$ and $\chi$ carry different irreps of $G_{\mathrm{HC}}$ and thus give rise to two separate cosets $\left(G_{\mathrm{EW}} / H_{\mathrm{EW}}\right) \times\left(G_{\mathrm{QCD}} / H_{\mathrm{QCD}}\right)$ both of the previous form. Let us pick one example for illustration purpose: the $G_{\mathrm{HC}}=S p(4)$ theory of [28]. To have a trilinear $G_{\mathrm{HC}}$ invariant while preserving asymptotic freedom, forces one to choose a combination of two fundamental $\mathbf{F}$ and one (two-index) antisymmetric $\mathbf{A}_{2}$ irreps.

For $S p(4), \mathbf{F}$ and $\mathbf{A}_{2}$ correspond to a pseudo-real and real irrep, respectively. Denoting by $\psi_{\alpha}^{a}$ and $\chi_{\beta}^{A}$ the respective Weyl fermions, with flavor indices $a, b \ldots$ and $A, B \ldots$, Weyl indices $\alpha, \beta \ldots$, and hypercolor index understood, we can build the following $S p(4)$ invariants: $\left(\psi_{\alpha}^{a} \chi_{\beta}^{A} \psi_{\gamma}^{b}\right),\left(\psi_{\dot{\alpha} a}^{\dagger} \chi_{\beta}^{A} \psi_{\dot{\gamma} b}^{\dagger}\right),\left(\psi_{\dot{\alpha} a}^{\dagger} \chi_{\dot{\beta} A}^{\dagger} \psi_{\gamma}^{b}\right)$. There are three more operators having an odd number of daggered (charge-conjugate) fermions combining with the previous ones to create a Dirac fermion from the vacuum. The round brackets (..) denote the unique $G_{\mathrm{HC}}$ invariant combination of hypercolor indices.

The fact that both $G_{\mathrm{HC}}$ irreps are self-conjugate means that we can take charge conjugation with impunity as far as the hypercolor indices are concerned and construct invariants in both cases, e.g., (considering now only the hypercolor indices and dropping the others) $(\psi \chi \psi)=\psi^{i} \chi^{[j k]} \psi^{l} \epsilon_{i j} \epsilon_{k l},\left(\psi^{\dagger} \chi^{\dagger} \psi\right)=\psi_{i}^{\dagger} \chi_{[j k]}^{\dagger} \psi^{k} \epsilon^{i j}$, and $\left(\psi^{\dagger} \chi \psi^{\dagger}\right)=\psi_{i}^{\dagger} \chi^{[i j]} \psi_{j}^{\dagger}$, where $\epsilon$ is the $S p(4)$ antisymmetric invariant tensor. Partners to the SM quarks must correspond to spin $1 / 2$ operators, so we must contract two Weyl indices of the same type. For the second and third operator, there is only one option $\left(\psi_{\dot{\alpha} a}^{\dagger} \chi_{\beta}^{A} \psi_{c}^{\dagger \dot{\alpha}}\right)$, $\left(\psi_{\dot{\alpha} a}^{\dagger} \chi_{A}^{\dagger \dot{\alpha}} \psi_{\gamma}^{c}\right)$. For the first operator, there are three seemingly different choices. However, two of them lead to the same invariant after taking into account the symmetry of the remaining indices and we can limit ourselves to $\left(\psi_{\alpha}^{a} \chi^{\alpha A} \psi_{\gamma}^{c}\right)$ and $\left(\psi_{\alpha}^{a} \chi_{\beta}^{A} \psi^{\alpha c}\right)$.

Having completed the treatment of the hypercolor and Weyl indices, we can simply read-off the symmetry properties of the global indices $a$ and $A$ that determine the SM quantum numbers of these operators. In the model [28], there are four spinors $\psi(a=1 \ldots 4)$ on which the custodial $S U(2)_{L} \times S U(2)_{R}$ acts block diagonally. From the terms of type $\left(\psi^{a} \chi \psi^{b}\right)$ and $\left(\psi_{a}^{\dagger} \chi \psi_{b}^{\dagger}\right)$, only the antisymmetric combination survives, decomposing as $\mathbf{6} \rightarrow(\mathbf{2}, \mathbf{2})+2 \times(\mathbf{1}, \mathbf{1})$ providing an appropriate representation for the embedding of $Q_{L}^{3}$ as well as $t_{R}$. The remaining operator $\left(\psi_{a}^{\dagger} \chi^{\dagger} \psi^{b}\right)$ has components in both the singlet and the adjoint of $S U(4)$ and can also provide possible partners for both fields, since $\mathbf{1 5} \rightarrow 2 \times(\mathbf{2}, \mathbf{2})+(\mathbf{1}, \mathbf{1})+(\mathbf{1}, \mathbf{3})+(\mathbf{1}, \mathbf{3})$.

The color $S U(3)$ group is easily embedded into $S U(6)$ block diagonally by taking $\mathbf{6} \rightarrow \mathbf{3}+\overline{\mathbf{3}}$. One should notice that it is possible to chose to switch the embedding of the EW group and the color group. The construction of the invariants remains unchanged, but the quantum numbers of the operators under the SM gauge group are drastically modified.

Even restricting to a simple $G_{\mathrm{HC}}$, there are many solutions to the group theory problem [27]. A further dynamical assumption can however be used to weed out 
many models as we shall now discuss. (This reasoning is also applicable to the single coset models.)

One of the big challenges of all solutions to EW SSB via strongly coupled gauge theories is to generate a sufficiently large top quark mass. The problem is quite generic, since, in the absence of scalars, the first invariants that can be added to the lagrangian for that purpose are four-fermi interactions of mass dimension $4 \times(3 / 2)=6$. Let us denote by $\Psi$ the generic hyperfermions and ignore the details about contractions of spinor and gauge indices for a moment. There are two possible ways of generating a mass, either via a bilinear term in the SM quarks $\mathcal{L}_{2}=\lambda_{2} Q_{L}\left(\Psi^{2}\right) t_{R}$ or a linear one $\mathcal{L}_{1}=\lambda_{1} Q_{L}\left(\Psi^{3}\right)+\lambda_{1}^{\prime} t_{R}\left(\Psi^{3}\right)^{\prime}$. (The notation $\left(\Psi^{n}\right)$ denotes a generic $G_{\mathrm{HC}}$ invariant composite operator that may still carry some SM quantum numbers.)

Because the operators have the same dimension, the couplings $\lambda$ are suppressed by a factor $1 / \Lambda_{\mathrm{UV}}^{2}$ at the scale $\Lambda_{\mathrm{UV}}$ at which they are generated. This scale should be very large, presumably larger than the flavor scale $\approx 10^{4} \mathrm{TeV}$ to avoid flavor constraints. In order for either of the interaction to survive at low energy and give a top Yukawa coupling $\mathcal{O}(1)$, the strongly coupled theory must be nearly conformal $[34,35]$ in a large energy range $\Lambda_{\mathrm{IR}} \ll \Lambda_{\mathrm{UV}}$. To fix the ideas, one can take $\Lambda_{\mathrm{IR}}=4 \pi f \approx 10 \mathrm{TeV}$ as the scale in which the theory leaves the conformal regime (presumably by decoupling some matter fields).

The composite operators $\left(\Psi^{2}\right)[36]$ or $\left(\Psi^{3}\right),\left(\Psi^{3}\right)^{\prime}$ must have the appropriate scaling dimension to make the interactions $\mathcal{L}_{2}$ or $\mathcal{L}_{1}$ close to being marginal. In both cases, this amounts to an anomalous dimension $\gamma^{*} \approx-2$ for these operators, but there is a qualitative difference between the two cases.

In the case of the bilinear $\left(\Psi^{2}\right)$, an anomalous dimension of -2 brings the operator dimension down to the free theory limit $(3-2=1)$. Therefore, in this case, we must have $\gamma^{*} \geq-2$ and the interaction can become marginal at best. In addition, bounds from conformal bootstrap [37] show that for $\left(\Psi^{2}\right)$ close to the free theory limit, the SM singlet $\left(\Psi^{2}\right)\left(\Psi^{2}\right)$ is necessarily relevant, reintroducing the fine-tuning issue for this operator, which necessarily appears in the full lagrangian. (Think of it as the composite analog of the SM mass term $-\mu^{2} H^{\dagger} H$.) Both problems are avoided in the PC case, since the required scaling dimension of the trilinear operators $\left(\Psi^{3}\right)$ need only be $9 / 2-2=5 / 2$ which is safely above the free limit of $3 / 2$ for fermions. Similarly, the appearance of a composite operator such as $\left(\Psi^{3}\right)$, of scaling dimension $5 / 2$, does generate a relevant SM singlet.

It is not unreasonable to expect a top partner of anomalous dimension close to -2 . (Some estimates using perturbation theory are given in [38]. See [39] for the QCD-like case for which the anomalous dimensions tend to be smaller.) In all cases, however, this comes together with an even larger (negative) anomalous dimension for the bilinears. If this continues beyond perturbation theory, then the fine-tuning problem reappears. There could be some crossing of anomalous dimensions at finite coupling, but, until we have a non-perturbative estimate, no strong conclusion can be reached on whether the PC approach with hyperfermions really overcomes all the problems with conformal Technicolor. It would be extremely helpful to gain some insight on these issues from the lattice. Unfortunately, to our knowledge, no computation of an anomalous dimension for fermionic bound states of this type has been performed to this day, due to the intrinsic difficulties in simulating gauge theories with slowly running coupling constants.

To summarize, the above picture is that of a strongly coupled CFT in the energy interval $\left[\Lambda_{\mathrm{IR}}, \Lambda_{\mathrm{UV}}\right]$, exiting the conformal regime at energies $\approx \Lambda_{\mathrm{IR}}$, below which it confines and breaks chiral symmetry. In this picture, the fermionic content of the theory below $\Lambda_{\mathrm{IR}}$ must still be such that a viable SSB coset, interpolating fields for the Higgs and other pNGBs, as well as top partner resonances are still possible to construct.

Making some reasonable but qualitative assumptions on the position of the conformal window (or "house" in this case [40]) in [41,42], a set of 12 models that fulfill all the above requirements was extracted from the many more of [27]. They are shown in Table 1 . The division is of course not clear-cut given the lack of rigorous results about the location of the conformal region. For instance, the last model could be conformal, or a similar model with $G_{\mathrm{HC}}=S U(6)$ could be added. However, these models are representative of the type of phenomenology to be expected from these constructions.

The minimum number of hyperfermions $\chi$, charged under QCD, is fixed by the requirement to being able to gauge $S U(3)_{c}$ as a subgroup of the global symmetry. To do that, $S U(3)_{c}$ should be free of 't Hooft anomalies from the point of view of the hypercolor theory. This fixes the minimum number of Weyl hyperfermions of this type to be always 6 ( $3+3$ for complex irreps). For the complex irrep, the anomaly-free embedding is the diagonal embedding into $S U(3) \times S U(3)$. The anomalyfree embedding of $S U(3)_{c}$ into $S U(6)$ is explicitly realized as $\left(\chi^{1}, \chi^{2}, \chi^{3}, \tilde{\chi}^{1}, \tilde{\chi}^{2}, \tilde{\chi}^{3}\right)$ for a pseudo-real irrep of $G_{\mathrm{HC}}$ and $\left(\left(\chi^{1}+\tilde{\chi}^{1}\right), i\left(\chi^{1}-\tilde{\chi}^{1}\right),\left(\chi^{2}+\tilde{\chi}^{2}\right), i\left(\chi^{2}-\right.\right.$ $\left.\left.\tilde{\chi}^{2}\right),\left(\chi^{3}+\tilde{\chi}^{3}\right), i\left(\chi^{3}-\tilde{\chi}^{3}\right)\right)$ for a real one (Weyl and hypercolor indices suppressed).

In light of the possible applications to LH models and DM, one can ask how many fermions of type $\psi$ can be added to enlarge the minimal EW coset. The requirement of asymptotic freedom poses weak constraints on this number. However, requiring the theory to be outside of the conformal range by employing the estimate [40] $4 n_{\psi} l(\psi)+4 n_{\chi} l(\chi)-11 C(G)<0$ gives the maximum number of hyperfermions of type $\psi$ presented in Table 1 . In this estimate, $n_{\chi}=6$ always, $l$ is the index of the appropriate irrep and $C(G)$ is the second Casimir of the hypercolor group. One sees that it is not possible to construct the LH model with PC of [19] in this way, while satisfying the above bound. Very recently, a model in which the color group is enlarged instead was presented in [31].

One of the advantages of having an underlying gauge theory is the possibility of making some edu- 
Table 1 Models of $\mathrm{CH}+\mathrm{PC}$ that are likely to lie outside of the conformal window

\begin{tabular}{|c|c|c|c|c|c|}
\hline Name & $G_{\mathrm{HC}}$ & $\psi$ & $\chi$ & $n_{\psi} \max$ & $G / H$ \\
\hline M1 & $S O(7)$ & $5 \times \mathbf{F}$ & $6 \times \operatorname{Spin}$ & 7 & $\frac{S U(5)}{S O(5)} \frac{S U(6)}{S O(6)} U(1)$ \\
\hline M2 & $S O(9)$ & $5 \times \mathbf{F}$ & $6 \times \operatorname{Spin}$ & 7 & \\
\hline M3 & $S O(7)$ & $5 \times$ Spin & $6 \times \mathbf{F}$ & 7 & \\
\hline M4 & $S O(9)$ & $5 \times$ Spin & $6 \times \mathbf{F}$ & 6 & \\
\hline M5 & $S p(4)$ & $5 \times \mathbf{A}_{2}$ & $6 \times \mathbf{F}$ & 5 & $\frac{S U(5)}{S O(5)} \frac{S U(6)}{S p(6)} U(1)$ \\
\hline M6 & $S U(4)$ & $5 \times \mathbf{A}_{2}$ & $3 \times(\mathbf{F}, \overline{\mathbf{F}})$ & 8 & $\frac{S U(5)}{S O(5)} \frac{S U(3) \times S U(3)^{\prime}}{S U(3)_{D}} U(1)$ \\
\hline M7 & $S O(10)$ & $5 \times \mathbf{F}$ & $3 \times(\mathbf{S p i n}, \overline{\mathbf{S p i n}})$ & 10 & \\
\hline M8 & $S p(4)$ & $4 \times \mathbf{F}$ & $6 \times \mathbf{A}_{2}$ & 4 & $\frac{S U(4)}{S p(4)} \frac{S U(6)}{S O(6)} U(1)$ \\
\hline M9 & $S O(11)$ & $4 \times$ Spin & $6 \times \mathbf{F}$ & 4 & \\
\hline M10 & $S O(10)$ & $4 \times(\mathbf{S p i n}, \overline{\mathbf{S p i n}})$ & $6 \times \mathbf{F}$ & 8 & $\frac{S U(4) \times S U(4)^{\prime}}{S U(4)_{D}} \frac{S U(6)}{S O(6)} U(1)$ \\
\hline M11 & $S U(4)$ & $4 \times(\mathbf{F}, \overline{\mathbf{F}})$ & $6 \times \mathbf{A}_{2}$ & 10 & \\
\hline M12 & $S U(5)$ & $4 \times(\mathbf{F}, \overline{\mathbf{F}})$ & $3 \times\left(\mathbf{A}_{2}, \overline{\mathbf{A}}_{2}\right)$ & 9 & $\frac{S U(4) \times S U(4)^{\prime}}{S U(4)_{D}} \frac{S U(3) \times S U(3)^{\prime}}{S U(3)_{D}} U(1)$ \\
\hline
\end{tabular}

In a impressive display of imaginative skills, these models are labeled M1...M12 in [42] and subsequent work. Model M8 corresponds to the one described in [28] and model M6 is discussed in [27]

cated assumptions on the nature of the spurion irreps into which the SM fermions are embedded (specifically the quarks of the third generation). A crucial requirement comes from the decay $Z \rightarrow b \vec{b}$, dominated by the LH current, forcing the following constraints on the quantum numbers of $Q_{L}^{3}$ under the custodial $S U(2)_{L} \times S U(2)_{R}[43]$ : either $T_{L}=T_{R}$ and $T_{L}^{3}=T_{R}^{3}$ or $T_{L}, T_{R}$ arbitrary and $T_{L}^{3}=T_{R}^{3}=0$. Interestingly, these are the type of irreps arising in this construction. For example, a possible theory based on a $G_{\mathrm{HC}}=S U(5)$ hypercolor group with four Dirac spinors $(\psi, \tilde{\psi})$ in the antisymmetric irrep $\mathbf{A}_{2}$ and three Dirac spinors $(\chi, \tilde{\chi})$ in the fundamental $\mathbf{F}$ would lead to top partners of type $\chi \psi \chi$, decomposing into the $(\mathbf{2}, \mathbf{1})+(\mathbf{1}, \mathbf{2})$ of $S U(2)_{L} \times S U(2)_{R}$ and violating the above condition. However, the model is (likely) inside of the conformal window, since $4 n_{\psi} l(\psi)+4 n_{\chi} l(\chi)-11 C(G)=10$ (counting $l(\mathbf{F})=1$ ) and thus excluded from Table 1 .

So far, we have taken for granted the fact that both the EW and the colored hyperfermions condense and give rise to each their own coset. However, having a model with fermionic bound states leads to the question of whether it is possible to have an alternative phase in which some of the symmetry is left unbroken and the 't Hooft anomaly is saturated by massless composite fermions [44]. Although there is solution to the anomaly matching condition for models of this type $[45,46]$, the results $[12,47]$ make it unlikely that the flavor symmetry contains an unbroken chiral component. Perhaps, the easiest way to argue is to notice that all fermionic bound states contain at least one hyperfermion of each type $\psi$ or $\chi$. Suppose we want to investigate the possibility that the global symmetry group associated with the $\chi \mathrm{s}$ is left unbroken, i.e., the $\chi \mathrm{s}$ do not condense. By giving a mass to the other type of fermion $\psi$ and invoking the persistent mass condition [47], we see that we can always give a mass to the fermionic composite states, and thus, the 't Hooft anomaly cannot be matched and the symmetry must be broken.
The presence of different irreps of fermions in these models has additional consequences that are of interest for phenomenology. One of the most basic ones is the presence of a (presumably very light) SM neutral pNGB arising from the anomaly-free $U(1)$ current $q_{\psi} \psi^{\dagger} \bar{\sigma}^{\mu} \psi+q_{\chi} \chi^{\dagger} \bar{\sigma}^{\mu} \chi$ for the appropriate values of the charges $q_{\psi, \chi}$. Its phenomenological signatures were investigated in $[42,48,49]$. The orthogonal combination of the currents is anomalous and its associated pseudoscalar acquires a larger mass in a similar way as the $\eta^{\prime}$ in QCD does. The appeal of having explicit underlying theories is that the anomaly coefficients can be computed explicitly and so can the couplings to gluons and EW bosons, after taking in due account the non-anomalous contributions from SM fermion loops, mostly the top.

\section{Phenomenological consequences}

The most generic prediction from any of these constructions is that the Higgs boson is accompanied by additional pNGBs. Indeed, the minimal coset $S O(5) / S O(4)$, referred to as the Minimal Composite Higgs Model (MCHM), is the only coset where no additional pNGBs arise. It has the virtue of providing a minimal setting $[25,50]$ in which to analyze the dynamics involved, but it does not follow the pattern of symmetry breaking most naturally realized in gauge theories with fermionic matter.

One can identify different classes of pNGBs in these models: First of all, we have neutral pNGBs that participate directly in the EWSB mechanism by acquiring a vev that breaks the EW symmetry, such as the $125 \mathrm{GeV}$ Higgs boson $h$ itself. These pNGBs are strongly constrained by the requirements of custodial symmetry. The numbers of possible custodial directions for the 
minimal three cosets are classified according to their $S U(2)_{L} \times S U(2)_{R}$ charges as

$$
\begin{array}{cl}
\text { For } S U(4) / S p(4): & h \in(\mathbf{2}, \mathbf{2}) \text { and } \eta \in(\mathbf{1}, \mathbf{1}) \\
\text { For } S U(5) / S O(5): & h \in(\mathbf{2}, \mathbf{2}), \Phi \in(\mathbf{3}, \mathbf{3})_{\mathrm{diag} .} \\
& \text { and } \eta \in(\mathbf{1}, \mathbf{1})
\end{array}
$$

For $S U(4) \times S U(4) / S U(4): h_{1,2} \in(\mathbf{2}, \mathbf{2})_{1,2}$ and $\eta \in(\mathbf{1}, \mathbf{1})$.

The generic pNGB $\xi$ associated with such directions include the $125 \mathrm{GeV}$ Higgs and develop couplings to the longitudinal EW bosons of type $\left(\kappa_{\xi} / v\right) \xi\left(m_{Z}^{2} Z_{\mu} Z^{\mu}+\right.$ $\left.m_{W}^{2} W_{\mu}^{+} W^{-\mu}\right)$ and are subjected to the regular Higgs searches, e.g., LEP bounds. (In the SM, $\xi=h$ and $\kappa_{h}=1$.) The remaining pNGBs should not develop a vev, or a very small one, but still couple quadratically to the EW bosons via covariant derivative terms of the form $\xi^{2} V^{\mu} V_{\mu}^{\prime}$ or linearly via anomalous terms that can also include the gluon. The expression for the neutral pNGBs pseudo-scalars is very familiar

$$
\begin{gathered}
\mathcal{L}=\frac{1}{16 \pi^{2} f} \xi\left(g^{2} \kappa_{W} W^{a \mu \nu} \tilde{W}_{\mu \nu}^{a}+g^{\prime 2} \kappa_{B} B^{\mu \nu} \tilde{B}_{\mu \nu}\right. \\
\left.\quad+g_{s}^{2} \kappa_{G} G^{A \mu \nu} \tilde{G}_{\mu \nu}^{A}\right)
\end{gathered}
$$

but the charged pNGBs can also have anomalous couplings. For instance [41], the doubly charged pNGB of the $S U(5) / S O(5)$ coset has a coupling of type $\xi^{--} W^{+\mu \nu} \tilde{W}_{\mu \nu}^{+}$. Interestingly, most of the anomalous couplings of the pNGBs of the $S U(4) \times S U(4) / S U(4)$ must vanish, because their $S U(2)_{L} \times S U(2)_{R}$ quantum numbers will not allow them. (Compare the $(\mathbf{3}, \mathbf{3})$ of $S U(5) / S O(5)$ with the $(\mathbf{3}, \mathbf{1})+(\mathbf{1}, \mathbf{3})$ of $S U(4) \times$ $S U(4) / S U(4)$.) This fact makes some of the latter pNGBs potentially very stable [21] provided that one can suppress the fermonic couplings, perhaps even providing DM candidates. (Another concrete possibility for DM candidates is to enlarge the coset, e.g., [51,52].)

In an underlying gauge theory, the strengths of the coefficients $\kappa_{W, B, G}$ are determined mostly by the ABJ anomaly of the hyperfermions, but they also get contributions from non-anomalous triangle loops with the SM fermions, just like the Higgs boson, if such coupling exists. Since the top quark is by far the one giving the largest contribution, to have $\kappa_{G}=0$ (which we could call "glue-phobic" behavior), we need both the ABJ anomaly and the couplings $\kappa_{\xi t} \xi \bar{t} t+\kappa_{\xi t}^{\prime} \xi \bar{t} \gamma^{5} t$ to vanish. This can happen naturally in some models for a particular choice of charges and spurions. Not only that, but a phenomenon occurring for some pNGBs in underlying theories with $S U(4) / S p(4)$ or $S U(4) \times S U(4) / S U(4)$ cosets is that the ABJ anomaly yields $\kappa_{W}+\kappa_{B}=0$, as well. This implies a "photo-phobic" behavior, i.e., the suppression of the coupling $\xi F^{\mu \nu} \tilde{F}_{\mu \nu}$ with the photon.

As already hinted in the previous paragraphs, we must also consider the couplings of the pNGBs to fermions. Here, the situation is more diverse, but we can still use the global charges as guidance. The first generic observation is that, in the cases where diagonal couplings are allowed, they will be proportional to the fermion mass and therefore will be mostly relevant to the third family of the SM. Only pNGBs that are doublets under $S U(2)_{L}$ are allowed to form Yukawa couplings with the SM fermions without the need of additional insertions. However, the larger symmetry of the particular coset under consideration can further suppress the couplings for some specific choices of spurions. A particularly interesting case is that of the singlet $\eta$ fully neutral under $S U(2)_{L} \times S U(2)_{R}$ whose coupling to fermions, particularly the top quark, can be dialed on and off by choosing different spurion irreps [53]. If fermio-phobic, such particle would be fiendishly difficult to detect, since it would be photo-phobic and gluephobic, as well $\left(\kappa_{W}+\kappa_{B}=\kappa_{G}=0\right)$. Perhaps, the best shot at a hadron collider is by pair production via an off-shell Higgs boson [54]. The situation for a lepton collider is discussed in [55].

It would be very useful to have more accurate estimates of the possible masses the composite states can have. Lattice gauge theory (to be reviewed elsewhere) can in principle provide such estimates, but the computations are quite demanding, particularly for the fermionic bound states requiring two different irreps of hyperfermions. Some non-perturbative estimates have also been performed [56] in the case of the $S U(4) / S p(4)$ coset and could be generalized to the other cases.

The most general expectation about pNGBs is that those that are charged under electromagnetism or color ought to be heavier than their neutral counterpart, since they acquire a loop-induced mass contribution of the order $N g \Lambda / 4 \pi$, with $g=e, g_{s}$ the appropriate coupling, $N=3,3 \times 8$ the number of d.o.f. of the gauge boson involved and $\Lambda$ is the typical confining scale of the hypercolor theory. Having knowledge of the masses of the lowest lying resonances would allow for a more precise estimate as in the case of the electromagnetic mass shift of the pion [57]. The better estimate in [56] finds $m \gtrsim 1.5 f$ for the gluon loop, roughly compatible with the naive estimate here.

\section{Conclusions}

We gave an overview of recent developments of BSM physics that can be constructed via a strongly coupled hypercolor gauge theory in four dimensions with only fermionic matter. We saw that there are many possible variations on this theme. Perhaps, the most substantial is whether we want to construct top partners from the underlying theory as well to implement the PC mechanism. This, together with the other requirements such as custodial symmetry and lack of conformal invariance in the IR, puts strong constraints on the matter content of these models and has some very specific predictions about the dynamics of the pNGBs. One could also consider enlarging the EW coset to build LH models, with our without PC.

The main theoretical hurdle in all of these constructions, just as it was for the original TC models, is to 
construct an "extended" dynamics mimicking that of extended Technicolor (ETC) $[1,4]$ giving rise to the appropriate four-fermi couplings at the scale $\Lambda_{\mathrm{UV}}$. (See [58] for recent ideas in this direction.) However, already below $\Lambda_{\mathrm{UV}}$, there is a variety of underlying gauge theories and related phenomenological signatures worth investigating. This richness of models should not make us believe that the situation has been fully charted. In fact, I dare propose the following conjecture: If some type of compositeness is at work above the EW scale, it will be described by a model with new ingredients that no one has yet thought about.

This work is supported in part by the Knut and Alice Wallenberg foundation under the grant KAW 2017.0100 (SHIFT project).

Funding Open access funding provided by Chalmers University of Technology.

Open Access This article is licensed under a Creative Commons Attribution 4.0 International License, which permits use, sharing, adaptation, distribution and reproduction in any medium or format, as long as you give appropriate credit to the original author(s) and the source, provide a link to the Creative Commons licence, and indicate if changes were made. The images or other third party material in this article are included in the article's Creative Commons licence, unless indicated otherwise in a credit line to the material. If material is not included in the article's Creative Commons licence and your intended use is not permitted by statutory regulation or exceeds the permitted use, you will need to obtain permission directly from the copyright holder. To view a copy of this licence, visit http://creativecomm ons.org/licenses/by/4.0/.

\section{References}

1. S. Dimopoulos, L. Susskind, Nucl. Phys. B 155, 237-252 (1979)

2. L. Susskind, Phys. Rev. D 20, 2619-2625 (1979)

3. S. Weinberg, Phys. Rev. D 13, 974-996 (1976)

4. E. Eichten, K.D. Lane, Phys. Lett. B 90, 125-130 (1980)

5. S. Dimopoulos, J.R. Ellis, Nucl. Phys. B 182, 505-528 (1982)

6. E. Farhi, L. Susskind, Phys. Rept. 74, 277 (1981)

7. H. Terazawa, K. Akama, Y. Chikashige, Phys. Rev. D 15, 480 (1977)

8. D.B. Kaplan, H. Georgi, Phys. Lett. B 136, 183-186 (1984)

9. M. Schmaltz and D. Tucker-Smith, Ann. Rev. Nucl. Part. Sci. 55 (2005), 229-270 arXiv:hep-ph/0502182 [hep-ph]

10. M.E. Peskin, Nucl. Phys. B 175, 197-233 (1980)

11. J. Preskill, Nucl. Phys. B 177, 21-59 (1981)

12. C. Vafa, E. Witten, Nucl. Phys. B 234, 173-188 (1984)

13. P. Sikivie, L. Susskind, M.B. Voloshin, V.I. Zakharov, Nucl. Phys. B 173, 189-207 (1980)

14. S. Weinberg, Phys. Rev. Lett. 19, 1264-1266 (1967)

15. J. Mrazek, A. Pomarol, R. Rattazzi, M. Redi, J. Serra, A. Wulzer, Nucl. Phys. B 853, 1-48 (2011). arXiv:1105.5403 [hep-ph]
16. E. Witten, Phys. Rev. Lett. 51, 2351 (1983)

17. M.J. Dugan, H. Georgi, D.B. Kaplan, Nucl. Phys. B 254, 299-326 (1985)

18. N. Arkani-Hamed, A. Cohen, E. Katz and A. Nelson, JHEP 07 (2002), 034 arXiv:hep-ph/0206021 [hep-ph]

19. I. Low, W. Skiba, D. Tucker-Smith, Phys. Rev. D 66 , 072001 (2002). arXiv:hep-ph/0207243 [hep-ph]

20. N. Arkani-Hamed, A. Cohen, E. Katz, A. Nelson, T. Gregoire and J. G. Wacker, JHEP 08 (2002), 021 arXiv:hep-ph/0206020 [hep-ph]

21. T. Ma, G. Cacciapaglia, JHEP 03, 211 (2016). arXiv:1508.07014 [hep-ph]

22. G. Cacciapaglia, F. Sannino, Phys. Lett. B 755, 328-331 (2016). arXiv:1508.00016 [hep-ph]

23. G. Cacciapaglia, S. Vatani and Z. W. Wang, arXiv:1909.08628 [hep-ph]

24. R. Contino, Y. Nomura and A. Pomarol, Nucl. Phys. B 671 (2003), 148-174 arXiv:hep-ph/0306259 [hep-ph]

25. K. Agashe, R. Contino and A. Pomarol, Nucl. Phys. B 719 (2005), 165-187 arXiv:hep-ph/0412089 [hep-ph]

26. D.B. Kaplan, Nucl. Phys. B 365, 259-278 (1991)

27. G. Ferretti, D. Karateev, JHEP 03, 077 (2014). arXiv:1312.5330 [hep-ph]

28. J. Barnard, T. Gherghetta, T.S. Ray, JHEP 02, 002 (2014). arXiv:1311.6562 [hep-ph]

29. G. Ferretti, JHEP 06, 142 (2014). arXiv:1404.7137 [hep$\mathrm{ph}]$

30. L. Vecchi, JHEP 02, 094 (2017). arXiv:1506.00623 [hep$\mathrm{ph}]$

31. T. Gherghetta, M.D. Nguyen, JHEP 12, 094 (2020). arXiv:2007.10875 [hep-ph]

32. M. Frigerio, J. Serra, A. Varagnolo, JHEP 06, 029 (2011). arXiv:1103.2997 [hep-ph]

33. D. Elander, M. Frigerio, M. Knecht and J. L. Kneur, arXiv:2011.03003 [hep-ph]

34. B. Holdom, Phys. Rev. D 24, 1441 (1981)

35. T.W. Appelquist, D. Karabali, L. Wijewardhana, Phys. Rev. Lett. 57, 957 (1986)

36. M. A. Luty and T. Okui, JHEP 09 (2006), 070 arXiv:hep-ph/0409274 [hep-ph]

37. R. Rattazzi, V.S. Rychkov, E. Tonni, A. Vichi, JHEP 12, 031 (2008). arXiv:0807.0004 [hep-th]

38. D. Buarque Franzosi and G. Ferretti, SciPost Phys. 7 (2019) no.3, 027 arXiv:1905.08273 [hep-ph]

39. C. Pica and F. Sannino, Phys. Rev. D 94 (2016) no.7, 071702 arXiv:1604.02572 [hep-ph]

40. T.A. Ryttov, F. Sannino, Int. J. Mod. Phys. A 25, 46034621 (2010). arXiv:0906.0307 [hep-ph]

41. G. Ferretti, JHEP 06, 107 (2016). arXiv:1604.06467 [hep-ph]

42. A. Belyaev, G. Cacciapaglia, H. Cai, G. Ferretti, T. Flacke, A. Parolini, H. Serodio, JHEP 01, 094 (2017). arXiv:1610.06591 [hep-ph]

43. K. Agashe, R. Contino, L. Da Rold and A. Pomarol, Phys. Lett. B 641 (2006), 62-66 arXiv:hep-ph/0605341 [hep-ph]

44. G.. 't Hooft, NATO Sci. Ser. B 59, 135-157 (1980)

45. G. Cacciapaglia and A. Parolini, Phys. Rev. D 93 (2016) no.7, 071701 arXiv:1511.05163 [hep-ph]

46. H. Gertov, A.E. Nelson, A. Perko, D.G. Walker, JHEP 02, 181 (2019). arXiv:1901.10456 [hep-ph]

47. J. Preskill, S. Weinberg, Phys. Rev. D 24, 1059 (1981) 
48. G. Cacciapaglia, G. Ferretti, T. Flacke and H. Serodio, Eur. Phys. J. C 78 (2018) no.9, 724 arXiv:1710.11142 [hep-ph]

49. G. Cacciapaglia, G. Ferretti, T. Flacke, H. Serodio, Front. in Phys. 7, 22 (2019). arXiv:1902.06890 [hep-ph]

50. R. Contino, L. Da Rold, A. Pomarol, Phys. Rev. D 75, 055014 (2007). arXiv:hep-ph/0612048 [hep-ph]

51. T. Alanne, D. Buarque Franzosi, M. T. Frandsen and M. Rosenlyst, JHEP 12 (2018), 088 arXiv:1808.07515 [hep-ph]

52. H. Cai and G. Cacciapaglia, arXiv:2007.04338 [hep-ph]

53. B. Gripaios, A. Pomarol, F. Riva, J. Serra, JHEP 04, 070 (2009). arXiv:0902.1483 [hep-ph]
54. D. Buarque Franzosi, G. Ferretti, L. Huang and J. Shu, SciPost Phys. 9 (2020), 077 arXiv:2005.13578 [hep-ph]

55. A. S. Cornell, A. Deandrea, B. Fuks and L. Mason, Phys. Rev. D 102 (2020) no.3, 035030 arXiv:2004.09825 [hepph]

56. N. Bizot, M. Frigerio, M. Knecht and J. L. Kneur, Phys. Rev. D 95 (2017) no.7, 075006 arXiv:1610.09293 [hep$\mathrm{ph}]$

57. T. Das, G. Guralnik, V. Mathur, F. Low, J. Young, Phys. Rev. Lett. 18, 759-761 (1967)

58. G. Cacciapaglia, S. Vatani and C. Zhang, arXiv:2005.12302 [hep-ph] 